\title{
Meissner-like effect and conductivity of power-Maxwell holographic superconductors
}

\author{
Doa Hashemi Asl ${ }^{1}$ and Ahmad Sheykhi ${ }^{1,2,3, *}$ \\ ${ }^{1}$ Physics Department and Biruni Observatory, College of Sciences, Shiraz University, Shiraz 71454, Iran \\ ${ }^{2}$ Research Institute for Astronomy and Astrophysics of Maragha (RIAAM), \\ P.O. Box 55134-441 Maragha, Iran \\ ${ }^{3}$ Institut für Physik, Universität Oldenburg, Postfach 2503 D-26111 Oldenburg, Germany
}

(Received 31 October 2019; published 14 January 2020)

\begin{abstract}
The full description of a superconductor requires that it has an infinite de conductivity (or zero electrical resistivity) as well as expels the external magnetic fields. Thus, for any holographic superconductor which is dual to a real superconductor, it is necessary to examine, simultaneously, these two features based on the gauge/gravity duality. In this paper, we explore numerically these two aspects of the higher dimensional holographic superconductors, in the presence of a power-Maxwell electrodynamics as the gauge field. At first, we calculate the critical temperature, condensation, conductivity, and superconducting gap, in the absence of magnetic field and disclose the effects of both power parameter, $s$, as well as the spacetime dimensions, $d$, on this quantities. Then, we immerse the superconductor into an external magnetic field, $B$, and observe that with increasing the magnetic field, the starting point of condensation occurs at temperature less than the critical temperature, $T_{c}$, in the absence of magnetic field. This implies that at a fixed temperature, we can define a critical magnetic field, above which the critical temperature goes to zero which is similar to the Meissner effect in superconductor. In these indications, we also try to show the distinction of the conformal invariance of the power-Maxwell Lagrangian that occurs for $s=d / 4$.
\end{abstract}

DOI: 10.1103/PhysRevD.101.026012

\section{INTRODUCTION}

The gauge/gravity correspondence [1-3], provides an efficient tool to explore strongly coupled phenomena in the field theory where the perturbational methods are no longer available. A version of this duality is called anti-deSitter/ conformal field theory (AdS/CFT) correspondence, which has been applied for investigating various aspects of holographic superconductors, using a gravity dual in a $(d+1)$ dimensional bulk for a superconductor which is localized on the $d$-dimensional boundary of the bulk [4,5]. AdS/CFT suggested that an instability for a charged black hole to develop scalar hair is dual to a superconducting phase transition. The investigations on the holographic superconductors have arisen a lot of attention in the past years and many features of them such as the critical temperature $T_{c}$, order parameter, critical exponents, conductivity and their responses to the magnetic field have been widely explored in the literatures [6-8]. In these

\footnotetext{
*asheykhi@shirazu.ac.ir
}

Published by the American Physical Society under the terms of the Creative Commons Attribution 4.0 International license. Further distribution of this work must maintain attribution to the author(s) and the published article's title, journal citation, and DOI. Funded by SCOAP. studies most of the holographically dual descriptions for a superconductor are based on a model in which a simple Einstein-Maxwell theory is coupled to a charged scalar field [9-13]. The properties of holographic superconductors for a given black hole depend on the electromagnetic field which is coupled to the charged scalar field and the underlying theory of gravity. In this direction the studies on the holographic superconductors have been generalized to other gravity theories such as Gauss-Bonnet gravity [14-21]. Moreover, the effects of other electrodynamics such as Born-Infeld, exponential, logarithmic on the physical properties of holographic superconductors were also explored in [22-29]. Conductivity for the holographic superconductors were calculated in different dimensions and in the presence of various electrodynamics [30-36]. Effects of an external magnetic field on the holographic superconductors were investigated by employing analytical and numerical methods [5,37-44]. In most cases, the focus is on the probe limit of the holographic superconductor where the matter and scalar fields do not backreact on the spacetime metric. One of the important properties of superconductor, in addition to the infinite dc conductivity, is their response to an external magnetic field, a phenomena known as the Meissner effect.

In the present paper we would like to study several aspects of the higher dimensional holographic superconductors 
when the gauge field is in the form of the power-Maxwell Lagrangian. The power-Maxwell electrodynamics is an extension of the Maxwell electrodynamics which its action has the form [45]

$$
I_{m}=-\int d^{d} x \sqrt{-g}\left(F_{a b} F^{a b}\right)^{s},
$$

where $s$ is a positive integer, $F_{a b}=\partial_{a} A_{b}-\partial_{b} A_{a}$ is the electromagnetic field tensor and $A_{a}$ is the electromagnetic potential. In arbitrary dimensions, when the power is $s=d / 4$, the power-Maxwell Lagrangian becomes invariant under the conformal transformation $\left(g_{a b} \rightarrow \Omega^{2} g_{a b}\right.$ and $A_{a} \rightarrow A_{a}$ ) and the resulting energy-momentum tensor is traceless $[45,46]$. It was argued that the theory of the conformally invariant Maxwell field brings rich physics compared to the linear standard Maxwell field. Applying the analytical Sturm-Liouville eigenvalue problem and the numerical shooting method in the background of $d$-dimensional Schwarzschild AdS black hole, the behavior of the holographic superconductors have been explored both in the probe limit [47] and in the presence of backreaction [48]. In the context of Gauss-Bonnet black holes, analytical and numerical studies on the properties of the holographic superconductors with power-Maxwell electrodynamics have been investigated $[49,50]$. Using the analytical matching method, the upper critical magnetic field of holographic superconductor with conformally invariant power-Maxwell electrodynamics have been explored in [51]. It was argued that in the presence of magnetic field, the physically acceptable phase behavior of the holographic superconductor can be deduced only for $s=d / 4$, which guaranties the conformal invariance of the power-Maxwell Lagrangian. Till now, conductivity as well as the effects of the magnetic field on the properties of the higher dimensional power-Maxwell holographic superconductors have not been explored by using the numerical shooting method. The novelty of our work comes from the fact that we employ the numerical method for investigating the Meissner-like effects, while most studies on the effect of magnetic field is based on the analytical methods [51]. The purpose is to see whether, when the gauge field is in the power-Maxwell form, the physically acceptable phase behavior of the holographic superconductor in the presence of magnetic field can be deduced only for the conformal invariance power parameter, $s=d / 4$, as argued in [51], or it may admit reasonable behavior for other values of the power parameter $s$.

Our aim in this paper is to investigate the responses of the power-Maxwell holographic superconductors on the electric field as well as on the external magnetic field in all higher dimensions. As we expect the properties of the holographic superconductor is affected by the choice of the electrodynamics. We shall do our calculations for several values of the power parameter and also compare the results for the case of conformal invariant with other cases. Throughout this paper, we neglect the backreaction of the scalar field and the gauge field for simplicity, so we cannot see all of the signature physics of a superconductor. However, in the final step, in order to examine the full effects of the external magnetic field on the holographic superconductor, we consider the effects of backreaction of the magnetic field on the background geometry.

The rest of the paper is organized as follows. In the next section, we introduce the basic field equations of the $d$-dimensional holographic superconductor with powerMaxwell electrodynamics and employ the numerical shooting method to obtain a relation between the critical temperature $T_{c}$ and charge density $\rho$. We also calculate the values of the condensation operator and the critical exponent in this section. In Sec. III we study the holographic electrical conductivity of the system and reveal the response of the system to an external electric field. Finally, in the last Sec. IV, we study the effects of an external magnetic field and observe the Meissner-like effect.

\section{HIGHER DIMENSIONAL HOLOGRAPHIC SUPERCONDUCTOR WITH POWER-MAXWELL ELECTRODYNAMICS}

\section{A. The background}

We begin with the higher dimensional Einstein action coupled to a massive charged complex scalar field in the presence of power-Maxwell field with a negative cosmological constant,

$$
\begin{aligned}
S= & \int d^{d} x \sqrt{-g}\left[R-2 \Lambda+\beta(-\mathcal{F})^{s}\right. \\
& \left.-|\nabla \psi-i q A \psi|^{2}-m^{2}|\psi|^{2}\right],
\end{aligned}
$$

where $R$ is the Ricci scalar, and the $d$-dimensional cosmological constant is defined as $\Lambda=-(d-1)(d-2) / 2 l^{2}$, where $l$ is the AdS radius [52]. Here $\beta$ is a constant, $\mathcal{F}=$ $F_{a b} F^{a b}$ where $A_{a}$ and $\psi$ are respectively, gauge field and scalar field with charge $q$ and mass $m$. The power-Maxwell contribution to the action is given by $\beta(-\mathcal{F})^{s}$ with the power parameter $s$. When $s=1$ and $\beta=1 / 4$ it reduces to the Maxwell case. In this paper, without loss of generality, we keep $\beta=1 / 4$ and only change the power parameter $s$.

Varying action (2) with respect to the metric $g_{a b}$, the gauge field $A_{a}$ and the scalar field $\psi$ yields the following field equations

$$
\begin{aligned}
& R_{a c}-\frac{g_{a c} R}{2}+\Lambda g_{a c} \\
& =\frac{1}{8} g_{a c}(-\mathcal{F})^{s}+\frac{s}{2}(-\mathcal{F})^{s-1} F_{a d} F_{c}{ }^{d}-\frac{g_{a c}}{2} m^{2}|\psi|^{2} \\
& -\frac{g_{a c}}{2}|\nabla \psi-i q A \psi|^{2} \\
& \quad+\frac{1}{2}\left[\left(\nabla_{a} \psi-i q A_{a} \psi\right)\left(\nabla_{c} \psi^{*}+i q A_{c} \psi^{*}\right)+a \leftrightarrow c\right],
\end{aligned}
$$




$$
\begin{aligned}
& s \nabla^{a}\left[F_{a c}(-\mathcal{F})^{s-1}\right] \\
& =i q\left[\psi^{*}\left(\nabla_{c}-i q A_{c}\right) \psi-\psi\left(\nabla_{c}+i q A_{c}\right) \psi^{*}\right], \\
& \left(\nabla_{a}-i q A_{a}\right)\left(\nabla^{a}-i q A^{a}\right) \psi-m^{2} \psi=0 .
\end{aligned}
$$

In this section, we consider the limit where the scalar field $\psi$ and the gauge field $A_{a}$ do not backreact on the background geometry. Thus the solution for the background geometry we take is the $d$-dimensional AdS-Schwarzschild black hole which is described by the following metric

$$
d s^{2}=-f(r) d t^{2}+\frac{1}{f(r)} d r^{2}+r^{2} h_{i j} d x^{i} d x^{j},
$$

where $f(r)$ is given by

$$
f(r)=\frac{r^{2}}{l^{2}}-\frac{1}{r^{d-3}}\left(\frac{r_{+}^{d-1}}{l^{2}}\right),
$$

and $r_{+}$is the event horizon radius. As expected, by neglecting the backreaction of the matter fields on the background geometry, just the existence of the black hole and the AdS radius are sufficient for determining the metric function. The Hawking temperature, which will be interpreted as the temperature of the holographic superconductors, is given by

$$
T=\frac{f^{\prime}\left(r_{+}\right)}{4 \pi}=\frac{(d-1) r_{+}}{4 \pi l^{2}} .
$$

In order to simplify the field equations and following [4], we choose a particular form for the gauge field $A_{a}$ and scalar field $\psi$ which is based on the fact that we know that what is the minimum content needed to produce a gravitational dual to a superconductor. In this step, that we want to explain exclusively a superconductor that exhibits condensation below the critical temperature $T_{c}$ (and without any external electric or magnetic field), that is enough to turn on only $A_{t}$ component by introducing the scalar hair for the black hole as [4],

$$
A_{a} d x^{a}=\phi(r) d t, \quad \psi=\psi(r),
$$

which depend only on $r$ coordinate. Inserting metric (6), scalar and gauge fields (9) in the field equations (4) and (5), we can arrive at the following pair of coupled second order differential equations for the gauge and scalar fields

$$
\begin{aligned}
& \phi^{\prime \prime}(r)-\frac{d-2}{(1-2 s) r} \phi^{\prime}(r)+\frac{q^{2} \psi^{2}(r) \phi^{\prime(2-2 s)}(r)}{s 2^{s-2}(1-2 s) f(r)} \phi(r)=0, \\
& \psi^{\prime \prime}(r)+\left(\frac{f^{\prime}}{f}+\frac{d-2}{r}\right) \psi^{\prime}(r)+\left(\frac{q^{2} \phi^{2}(r)}{f^{2}(r)}-\frac{m^{2}}{f(r)}\right) \psi(r)=0,
\end{aligned}
$$

with appropriate boundary conditions at the horizon and at the asymptotic infinity. We require at the horizon $\left(r=r_{+}\right)$,

$$
\phi\left(r_{+}\right)=0, \quad \psi\left(r_{+}\right)=\frac{f^{\prime}\left(r_{+}\right) \psi^{\prime}\left(r_{+}\right)}{m^{2}} .
$$

While at the boundary $(r \rightarrow \infty)$, the solutions behave like

$$
\begin{aligned}
& \phi(r)=\mu-\frac{\rho^{\frac{1}{2 s-1}}}{r^{\frac{d-2}{2 s-1}-1}}, \\
& \psi(r)=\frac{\psi_{-}}{r^{\Delta_{-}}}+\frac{\psi_{+}}{r^{\Delta_{+}}},
\end{aligned}
$$

where $\mu$ and $\rho$ are, respectively, the chemical potential and charge density of dual field theory, and

$$
\Delta_{ \pm}=\frac{1}{2}\left[(d-1) \pm \sqrt{(d-1)^{2}+4 m^{2} l^{2}}\right] .
$$

In contrast to other electrodynamics, in case of the powerMaxwell electrodynamics, the asymptotic behavior of $\phi$ depends on the electrodynamics parameter $s$, and since the gauge field should have a finite value at the boundary $(r \rightarrow \infty)$, we may arrive a restriction on the value of $s$. The meaningful range of the parameter $s$ is obtained $1 / 2<$ $s<(d-1) / 2$ [49], thus at any dimension, we make our choices for power parameter with respect to this range.

The coefficients $\psi_{-}$and $\psi_{+}$both multiply normalizable modes of the scalar field equations and according to the AdS/CFT correspondence, they correspond to the expectation value of condensation operator on the boundary [4]. Indeed, either $\psi_{+}$or $\psi_{-}$can be dual to the value of the condensation operator, and the other one is dual to its source. Following [9], we can impose the boundary condition in which either $\psi_{-}$or $\psi_{+}$vanishes. In this paper we set $\psi_{-}=0$. One can solve the field equations (10) and (11) by different methods. The method we choose for this purpose is a numerical shooting method. At first, we make the coordinate transformation as $r \rightarrow z=r_{+} / r$ in the equations and work with dimensionless parameter $z$. We also assume the values of $q, r_{+}$and $l$ to be unity according to the symmetries. In addition, we make our choices for the values of the parameters, in the allowed ranges. In this regards, $\mathrm{m}^{2}$ must satisfy the BF (Breitenlohner-Freedman) bound [52], which in our case is given by $m^{2} \geq-(d-1)^{2} / 4 l^{2}$.

\section{B. Critical temperature}

Let us start by deriving the critical temperature, $T_{c}$, of the higher dimensional holographic superconductor in the presence of power-Maxwell electrodynamics. Our investigation will be followed by the method developed in [32]. In this calculation we also use the charge density $\rho$ to fix a scale. From QFT dimensional analysis, for the powerMaxwell Lagrangian in a $d$-dimensional AdS bulk, we find that the dimension of any component of gauge field $A_{a}$ is 
TABLE I. Critical temperature $T_{c}$ for $d=4,5$, and 6 with $\Delta_{+}=2,3$, and 4 .

\begin{tabular}{lccc}
\hline \hline$s$ & $T_{c}(d=4)$ & $T_{c}(d=5)$ & $T_{c}(d=6)$ \\
\hline $3 / 4$ & $0.194 \rho^{1 / 2}$ & $\ldots$ & $\ldots$ \\
1 & $0.118 \rho^{1 / 2}$ & $0.198 \rho^{1 / 3}$ & $0.271 \rho^{1 / 4}$ \\
$5 / 4$ & $0.045 \rho^{1 / 2}$ & $0.128 \rho^{1 / 3}$ & $0.202 \rho^{1 / 4}$ \\
$6 / 4$ & $\cdots$ & $0.068 \rho^{1 / 3}$ & $0.138 \rho^{1 / 4}$ \\
$7 / 4$ & $\cdots$ & $\cdots$ & $0.085 \rho^{1 / 4}$ \\
\hline \hline
\end{tabular}

always one, independent of the power parameter $s$. Thus, from Eq. (13), the dimension of charge density is obtained $(d-2)$, regardless of the value of the power parameter $s$. Since the dimension of the temperature is one, therefore in order to have the dimensionless quantities, one should consider $\rho^{1 /(d-2)}$. In Tables I, we summarize the results of the critical temperature for different values of $s$ and $d$ with boundary condition $\psi_{-}=0$ and the mass of scalar field $m^{2}=-2,-3$ and -4 for $d=4,5$ and 6 .

From this table, we find that with increasing the power parameter $s$, the critical temperature will be decreased as well, which implies that the condensation is harder to form. Moreover, the results are also consistent with the general understanding that the phase transition is easier to achieve in higher dimensional systems. For completeness, in this table, besides the Maxwell case where $s=1$, in any dimension $d$, we choose three values for the power parameter $s$, namely the conformal invariance case, $s=d / 4$, the case $s<d / 4$ and the case $s>d / 4$.

\section{Condensation operator and critical exponent}

The first expected result in the study of a holographic superconductor is quickly increase of the order parameter by reaching critical temperature $T_{c}$. Here the order parameter is a charged operator in the boundary that is related by AdS/CFT to the coefficients of the asymptotic behavior of the scalar field according to Eq. (14), which based on our choice $\left(\psi_{-}=0\right), \psi_{+}$gives us the expectation value of the condensation operator [5]. Solving the field equations, we obtain $\psi_{+}$(and thus $\left\langle\mathcal{O}_{+}\right\rangle$) values. Using the Lagrangian
TABLE II. The values of $\gamma$ for $d=4,5$, and 6 .

\begin{tabular}{lccr}
\hline \hline$s$ & $\sqrt{2} \gamma(d=4)$ & $\gamma(d=5)$ & $\gamma(d=6)$ \\
\hline $3 / 4$ & 57.3 & $\ldots$ & $\cdots$ \\
1 & 137.3 & 384.4 & 942.2 \\
$5 / 4$ & 255.5 & 896.3 & 2593.5 \\
$6 / 4$ & $\cdots$ & 1830.4 & 6199.9 \\
$7 / 4$ & $\cdots$ & $\cdots$ & 13549.2 \\
\hline \hline
\end{tabular}

dimensional analysis, we obtain $\Delta+$ as the dimension of $\psi_{+}$, and hence the dimension of condensation operator will be the same. So it is easy to make dimensionless parameters for the plotting. we plot for three dimensions of spacetime: $d=4,5$, and 6 and at any dimension for three values of power parameter $s$.

As seen from the figures, the values of the order parameter becomes nonzero at $T=T_{c}$ and grow up quickly when $T \rightarrow 0$. This means the charged scalar operator has condensed. Moreover, from the theory of superconductor [53], near the critical temperature $\left(T \sim T_{c}\right)$, as the critical temperature approaches from below, we find the order parameter as

$$
\left\langle\mathcal{O}_{+}\right\rangle=\gamma T_{c}^{\Delta_{+}}\left(1-\frac{T}{T_{c}}\right)^{1 / 2}
$$

where $\gamma$ is a constant and $1 / 2$ is the critical exponent for the second order phase transition. Also from analytical methods (Sturm-Liouville or matching method), this result was obtained in earlier studies, and except the coefficient $\gamma$, the form of this relation is in a general form that do not depend on the choice of the electrodynamics and the dimension of the spacetime. Therefore, it is interesting to fit the plots we obtained from the numerical shooting method for the holographic superconductor, on the formula like (16) and calculate the coefficient $\gamma$ and critical exponent. The results are summarized in Table II.

Besides, for all values of the power parameter $s$ and $d$, we obtain a value of about $1 / 2$ for the critical exponent. From Table II and Fig. 1, we can see that in higher

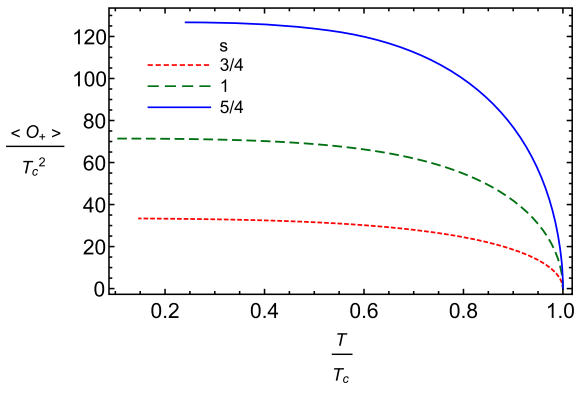

(a) $d=4$

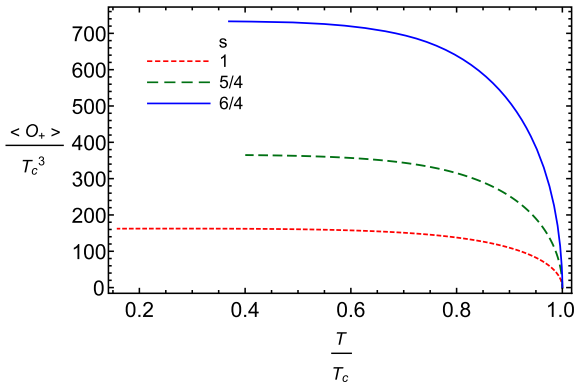

(b) $d=5$

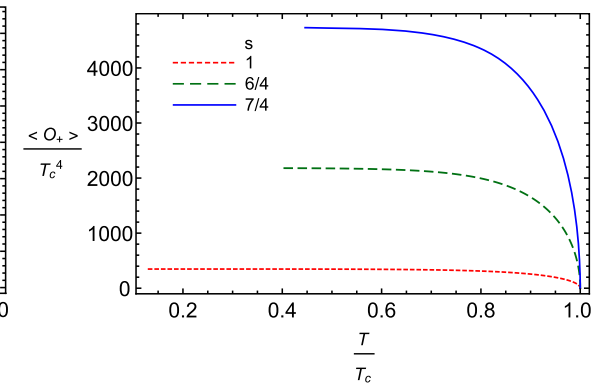

(c) $d=6$

FIG. 1. Dimensionless condensation operator $\left(\frac{\left\langle\mathcal{O}_{+}\right\rangle}{T_{c}^{\Delta_{+}}}\right)$versus dimensionless temperature $T / T_{c}$ for $d=4,5$, and 6 . 
dimensions and for larger value of $s, \gamma$ and (as a result) the rescaled condensation operator $\left(\frac{\left\langle\mathcal{O}_{+}\right\rangle}{T_{c}^{\Delta+}}\right)$ have explicitly increase, that arises from the fact that in these cases the critical temperature decreases. Until now, we have introduced and built a model of holographic superconductor in the presence of the power-Maxwell electrodynamics and observed the condensation for this kind of holographic superconductor. However, as we pointed out earlier, this study is not enough and the model can describe a real dual to superconductor if displays both properties of the infinite dc conductivity as well as expelling the magnetic field lines in the superconducting phase. So from now on, in order to study these two properties of this superconductor, in the first step, we apply an external electric field and in the second step, we apply an external magnetic field. In other words, we will examine the effects of background electric and magnetic fields on the holographic superconductor in the presence of the power-Maxwell electrodynamics. As we shall see the presence of this electrodynamics affects the conductivity and the way of expelling the applied magnetic field.

\section{CONDUCTIVITY}

In this section, we will apply an electric field in order to study the transport phenomena in the holographic superconductors with power-Maxwell field. Since the purpose is to obtain the dc and ac conductivity (that means conductivity in different frequency), we should consider the applied field to fluctuate relative to time with the frequency $\omega$ in the boundary. The result of illustrating the above concepts by employing AdS/CFT correspondence is considering fluctuation of the field $A_{x}$ in the bulk. This fluctuation is dual to the electric current $J^{x}$ operator in the CFT [4,5]. We take all the currents and sources in the $x$ direction. It should be noted that if we consider the backreaction of the matter fields on the spacetime geometry, we should also take into account the fluctuation of the $g_{t x}$ component of the metric. For simplicity, here we neglect the backreaction. Thus, we assume the Maxwell field as

$$
A_{a} d x^{a}=\phi(r) d t+A_{x}(r) e^{-i \omega t} d x .
$$

Our strategy is to obtain the field equation of $A_{x}(r)$ and try to solve it with appropriate boundary conditions in the bulk for different values of the power parameter and dimension of spacetime. Then, we shall use this solution to find out the current as well as the conductivity in the boundary. From Eq. (4) the field equation for $A_{x}(r)$ is given by

$$
\begin{gathered}
A_{x}^{\prime \prime}(r)+\left(\frac{2(s-1) \phi^{\prime \prime}(r)}{\phi^{\prime}(r)}+\frac{(d-4)}{r}+\frac{f^{\prime}(r)}{f(r)}\right) A_{x}^{\prime}(r) \\
+\left(\frac{\omega^{2}}{f^{2}(r)}-\frac{\psi^{2}(r)}{2^{(s-2)} s \phi^{(2 s-2)}(r) f(r)}\right) A_{x}(r)=0 .
\end{gathered}
$$

It should be noted that in this calculation we linearize the equation that means working in the regime of linear response. It is a matter of calculations to show that the asymptotic behavior of this equation for $r \rightarrow \infty$ is given by

$$
A_{x}^{\prime \prime}(r)+\frac{d-2}{(1-2 s) r} A_{x}^{\prime}(r)+\frac{\omega^{2}}{r^{4}} A_{x}(r)=0 .
$$

It is apparent that the solutions of this equation depend on the both $s$ and $d$ parameters. Therefore, we present the solution for several values of $d$ and $s$. The solution for $d=4$ is

$$
A_{x}= \begin{cases}A^{(0)}+\frac{\omega^{2} A^{(0)}}{2 r^{2}}+\frac{A^{(1)}}{r^{3}}+\ldots, & \text { for } s=3 / 4 \\ A^{(0)}+\frac{A^{(1)}}{r}-\frac{\omega^{2} A^{(0)}}{2 r^{2}}+\ldots, & \text { for } s=1 \\ A^{(0)}+\frac{A^{(1)}}{r^{1 / 3}}-\frac{3 \omega^{2} A^{(0)}}{10 r^{2}}+\frac{3 \omega^{2} A^{(1)}}{14 r^{7 / 3}}+\ldots, & \text { for } s=5 / 4\end{cases}
$$

For $d=5$ :

$$
A_{x}= \begin{cases}A^{(0)}+\frac{A^{(1)}}{r^{2}}+\frac{\omega^{2} A^{(0)} \ln (k r)}{2 r^{2}}+\ldots, & \text { for } s=1 \\ A^{(0)}+\frac{A^{(1)}}{r}-\frac{\omega^{2} A^{(0)}}{2 r^{2}}-\frac{\omega^{2} A^{(1)}}{6 r^{3}}+\ldots, & \text { for } s=5 / 4 \\ A^{(0)}+\frac{A^{(1)}}{r^{1 / 2}}-\frac{\omega^{2} A^{(0)}}{3 r^{2}}-\frac{\omega^{2} A^{(1)}}{5 r^{10 / 4}}+\ldots, & \text { for } s=6 / 4\end{cases}
$$

And for $d=6$ :

$$
A_{x}= \begin{cases}A^{(0)}+\frac{\omega^{2} A^{(0)}}{2 r^{2}}+\frac{A^{(1)}}{r^{3}}-\frac{\omega^{4} A^{(0)}}{8 r^{4}}-\frac{A^{(1)} \omega^{2}}{10 r^{5}}+\ldots, & \text { for } s=1, \\ A^{(0)}+\frac{A^{(1)}}{r}-\frac{\omega^{2} A^{(0)}}{2 r^{2}}-\frac{\omega^{2} A^{(1)}}{6 r^{3}}+\frac{\omega^{4} A^{(0)}}{24 r^{4}}-\frac{\omega^{4} A^{(1)}}{120 r^{5}}-\frac{\omega^{6} A^{(0)}}{720 r^{6}}+\ldots, & \text { for } s=6 / 4, \\ A^{(0)}+\frac{A^{(1)}}{r^{3 / 5}}-\frac{5 \omega^{2} A^{(0)}}{14 r^{2}}-\frac{5 \omega^{2} A^{(1)}}{26 r^{13 / 5}}+\frac{25 \omega^{4} A^{(0)}}{925 r^{4}}+\frac{25 \omega^{4} A^{(1)}}{2392 r^{23 / 5}}+\ldots, & \text { for } s=7 / 4,\end{cases}
$$

where $A^{(0)}, A^{(1)}$, and $k$ are constant parameters. Following Ref. [54], for calculating the boundary current operator, we first need the on shell action $S_{\text {o.s. }}$. Then one can use the following relation for calculating the current,

$$
J=\frac{\delta S_{\text {o.s. }}}{\delta A^{(0)}}
$$

The on shell action is given by [54] 


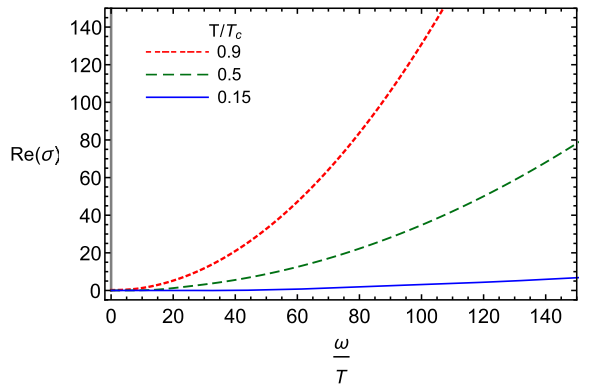

(a) $d=4, s=3 / 4$

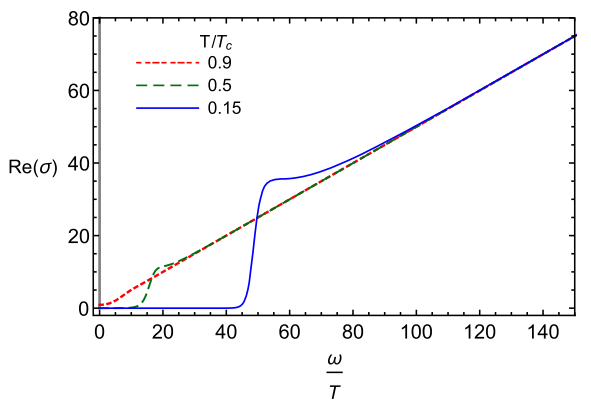

(d) $d=5, s=1$

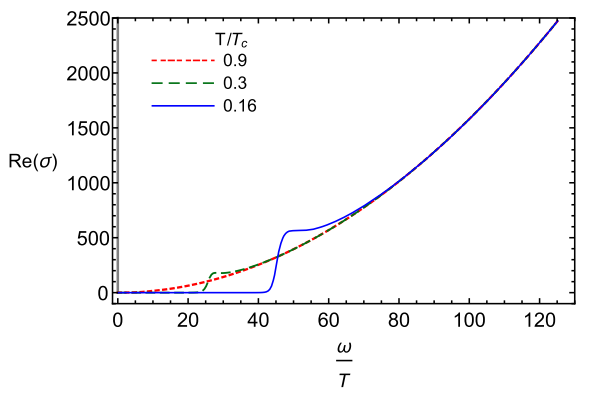

(g) $d=6, s=1$

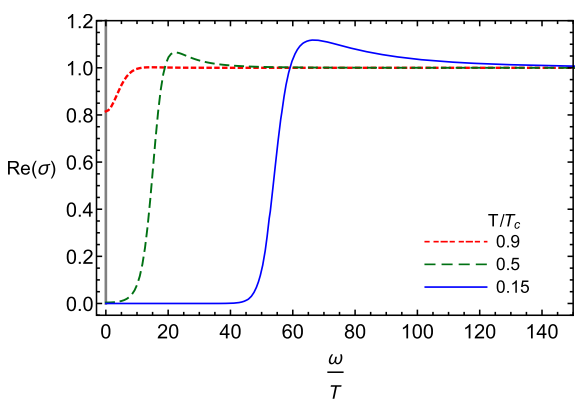

(b) $d=4, s=1$

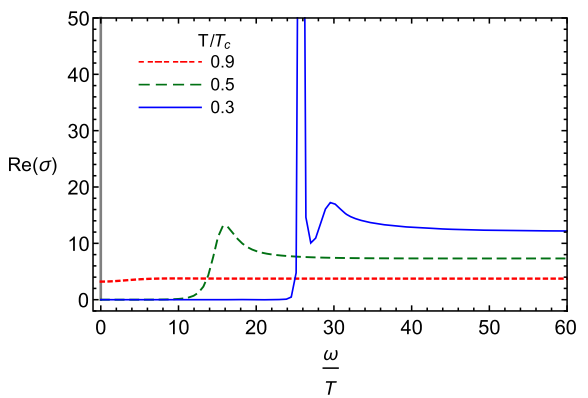

(e) $d=5, s=5 / 4$

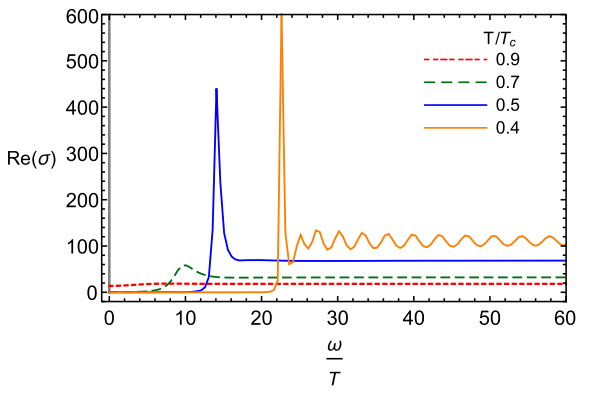

(h) $d=6, s=6 / 4$

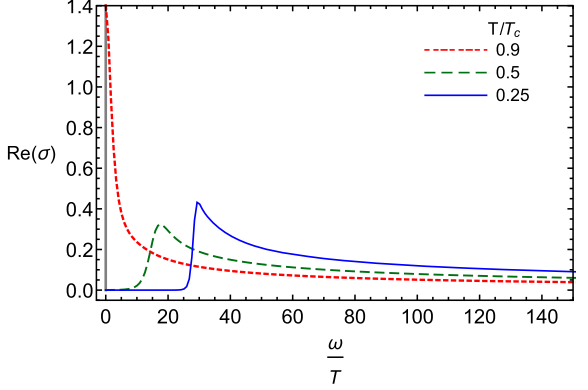

(c) $d=4, s=5 / 4$

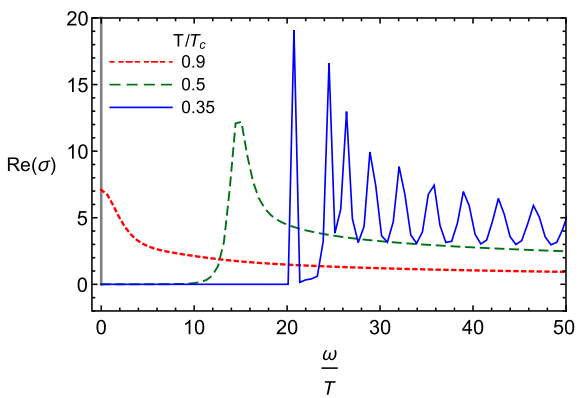

(f) $d=5, s=6 / 4$

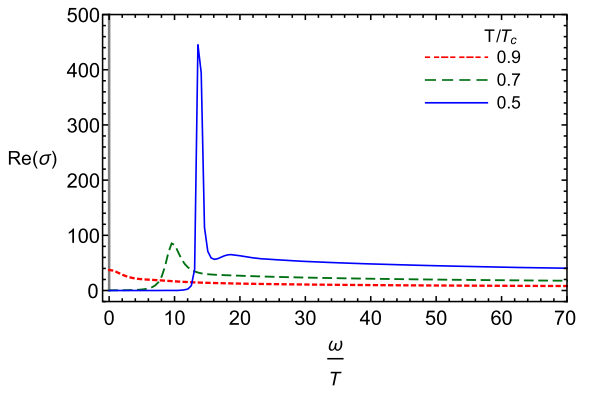

(i) $d=6, s=7 / 4$

FIG. 2. The real part of conductivity for different temperature in terms of $\omega / T$.

$$
S_{\text {o.s. }}=\int_{r_{+}}^{\infty} d r \int d^{d-1} x \sqrt{-g} \mathcal{L}
$$

where in our case it reduces to

$S_{\text {o.s. }}=\left.\int d^{d-1} x\left(-2^{(s-2)} s r^{d-4} f(r) \phi^{\prime(2 s-2)} A_{x}(r) A_{x}^{\prime}(r)\right)\right|_{r \rightarrow \infty}$.

Combining Eqs. (19), (20), (21), and (13) with Eq. (24), we obtain explicitly the on shell action for different values of the power parameter $s$ and for different spacetime dimension $d$. Then, employing Eq. (22), we can find the current for different values of $s$ and $d$. But most probably in this calculation, we may encounter with divergence terms in the action when we set $r \rightarrow \infty$. In order to remove the divergences in the action, we add a proper counterterm [55]. We need counterterms for $d=4$ and $s=3 / 4, d=5$, and $s=1, d=6$ and $s=1$. For the economic reasons we do not present here the counterterms. One can calculate the conductivity for the system from $\sigma_{x x}=J_{x} / E_{x}$, by having the applied electric field and the current caused by it. The electric field is $E_{x}=-\partial_{t} A_{x}=i \omega A^{(0)}$. Thus, for the conductivity we find out for $d=4$,

$$
\sigma= \begin{cases}\frac{3 \sqrt{3} A^{(1)}}{4 * 2^{1 / 4} i \omega \rho A^{(0)}}, & \text { for } s=3 / 4, \\ \frac{A^{(1)}}{i \omega A^{(0)}}, & \text { for } s=1, \\ \frac{5 A^{(1)} 1^{1 / 3}}{6 \sqrt{3} * 3^{3 / 4} i \omega A^{(0)}}, & \text { for } s=5 / 4 .\end{cases}
$$

For $d=5$ we find

$$
\sigma= \begin{cases}\frac{2 A^{(1)}}{i \omega A^{(0)}}+\frac{i \omega}{2}, & \text { for } s=1, \\ \frac{5 A^{(1)} \rho^{1 / 3}}{2 * 2^{3 / 4} i \omega A^{(0)}}, & \text { for } s=5 / 4, \\ \frac{3 A^{(1)} \rho^{1 / 2}}{4 \sqrt{2} i \omega A^{(0)}}, & \text { for } s=6 / 4 .\end{cases}
$$




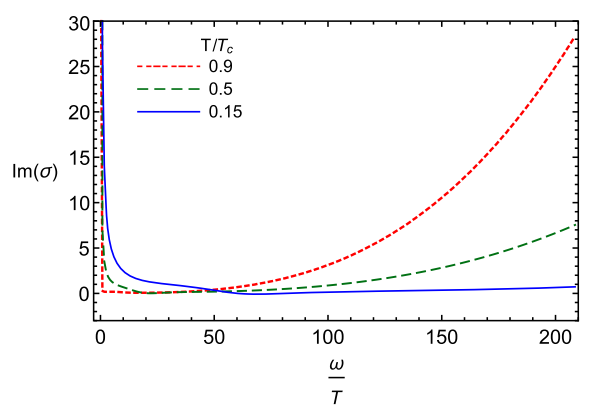

(a) $d=4, s=3 / 4$

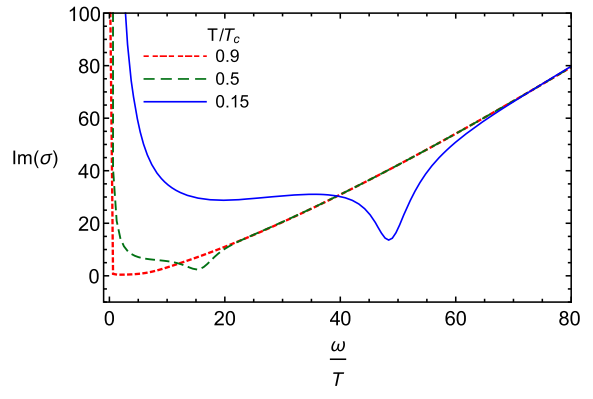

(d) $d=5, s=1$

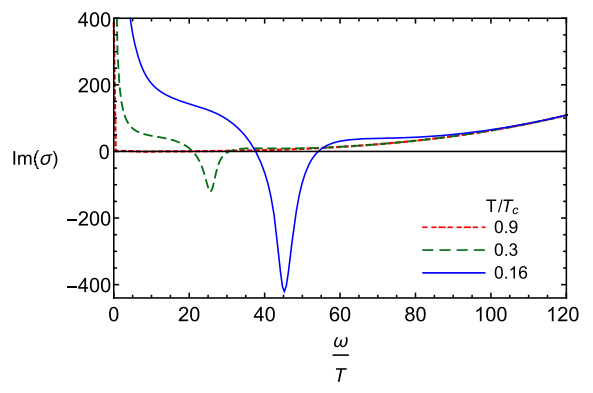

(g) $d=6, s=1$

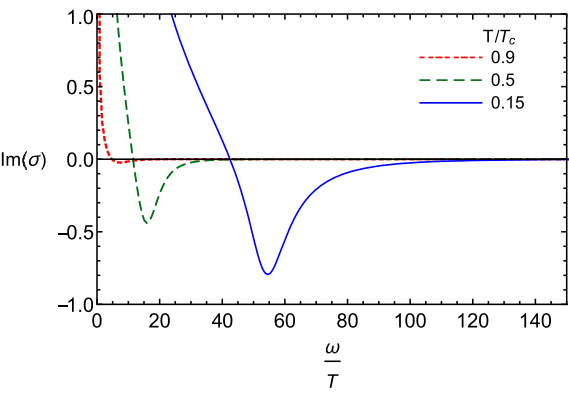

(b) $d=4, s=1$

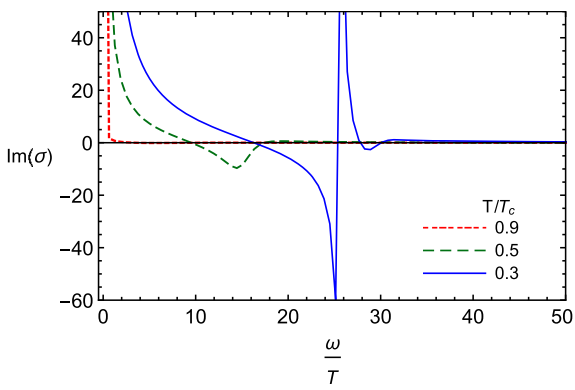

(e) $d=5, s=5 / 4$

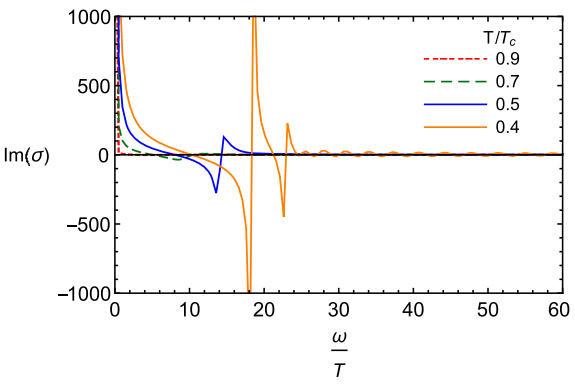

(h) $\quad d=6, s=6 / 4$

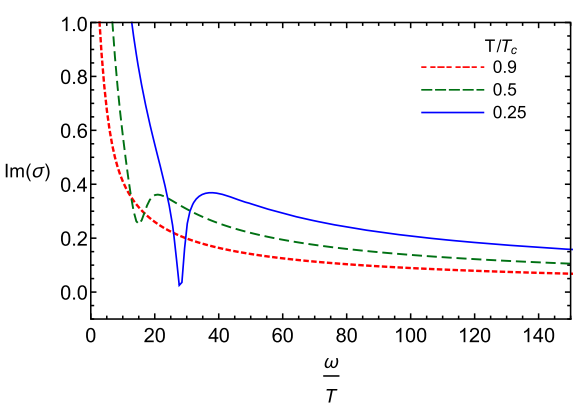

(c) $d=4, s=5 / 4$

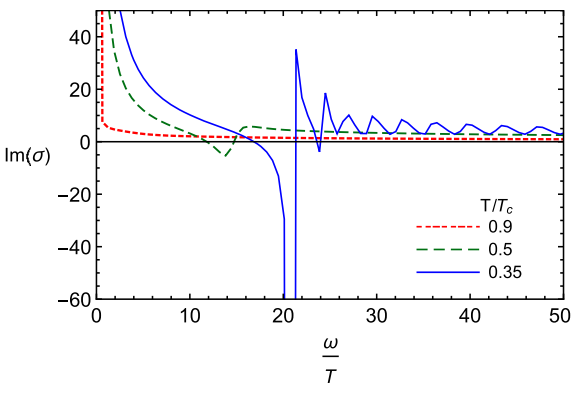

(f) $d=5, s=6 / 4$

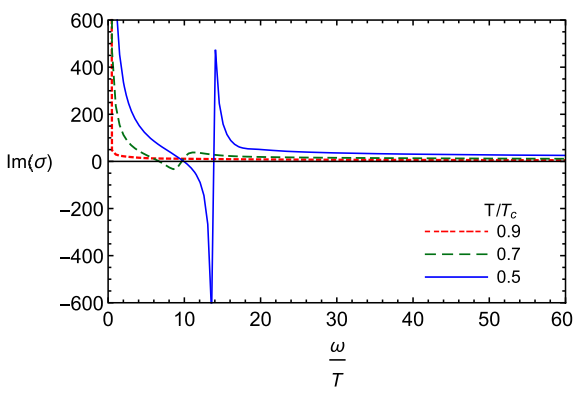

(i) $d=6, s=7 / 4$

FIG. 3. The imaginary part of conductivity for different temperature in terms of $\omega / T$.

Finally, for $d=6$, we obtain

$$
\sigma= \begin{cases}\frac{3 A^{(1)}}{i \omega A^{(0)}}, & \text { for } s=1, \\ \frac{3 A^{(1)} \rho^{1 / 2}}{\sqrt{2} i \omega A^{(0)}}, & \text { for } s=6 / 4, \\ \frac{63 \sqrt{3} A^{(1)} \rho^{3 / 5}}{50 \sqrt{5} * 2^{1 / 4} i \omega A^{(0)}}, & \text { for } s=7 / 4\end{cases}
$$

Let us note that the relations for $\sigma$, contain a power of $\rho$ for some values of $s$. This comes from the fact that Eq. (24), includes $\phi^{\prime(2 s-2)}$. Using Eq. (13) for $\phi$, which is a function of $\rho$ and $s$, the dependence of $\sigma$ on the charge density $\rho$ is expected. Next, we solve numerically the Eq. (18) in the bulk. Having at hand the behavior of the field $A_{x}(r)$ in the bulk, one can find its behavior at the boundary, that determines the values of $A^{(0)}$ and $A^{(1)}$. Thus, we can calculate the conductivity as a function of frequency $\omega$, for all supposing values of $d$ and $s$ from Eq. (25), (26), and (27).
It is also interesting to study the behavior of conductivity $\sigma$ for different values of $\omega$, and investigate the behavior of $\mathrm{dc}$ and ac conductivity for the holographic superconductor in the presence of power-Maxwell gauge field. The behavior of the real and imaginary parts of conductivity as a function of frequency for various power parameter $s$ and in various dimension, at different temperature, are depicted in Figs. 2 and 3. The first point that comes out from these plots, is the existence of a pole function in the imaginary parts of the conductivity at $\omega=0$ for all values of $s$ and $d$. This yields a delta function at $\omega=0$ for real part of conductivity, which represents the infinite dc conductivity for the holographic superconductor. For other value of $\omega$, we see that the superconducting gap appears and becomes deep with decreasing the temperature, which means the $\omega_{g}$ becomes larger. At enough large frequency, the conductivity for a fixed dimension of spacetime $d$, behaves as follows. For the conformal invariance powerMaxwell Lagrangian $(s=d / 4)$, the conductivity does not 


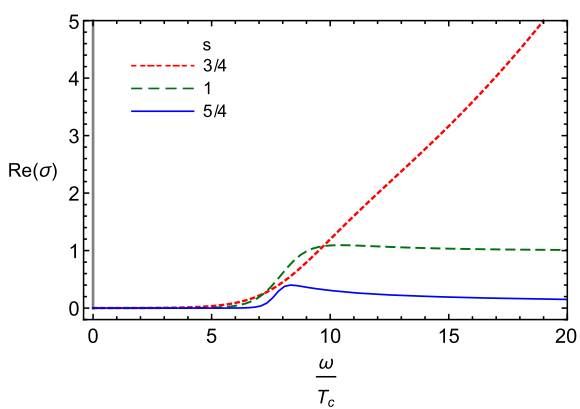

(a) $d=4, T \approx 0.3 T_{c}$

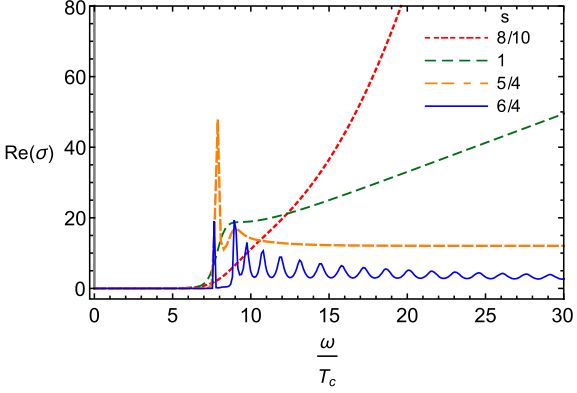

(b) $d=5, T / T_{c} \approx 0.3 T_{c}$

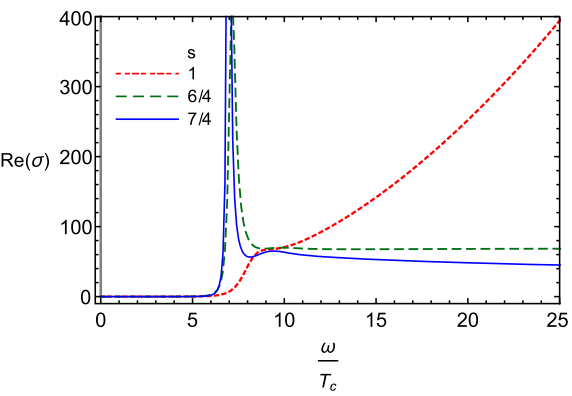

(c) $d=6, T / T_{c} \approx 0.5 T_{c}$

FIG. 4. The real part of conductivity for different $s$ in terms of $\omega / T_{c}$.

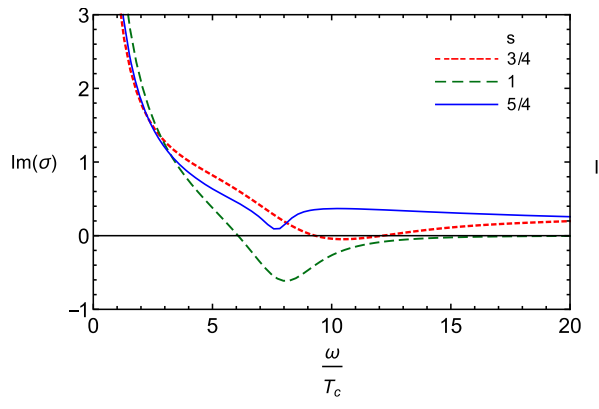

(a) $d=4, T \approx 0.3 T_{c}$

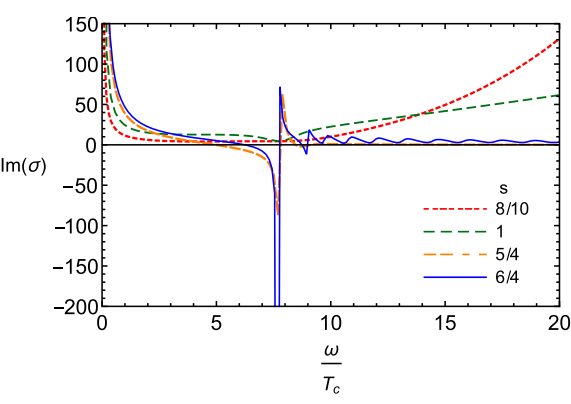

(b) $d=5, T / T_{c} \approx 0.3 T_{c}$

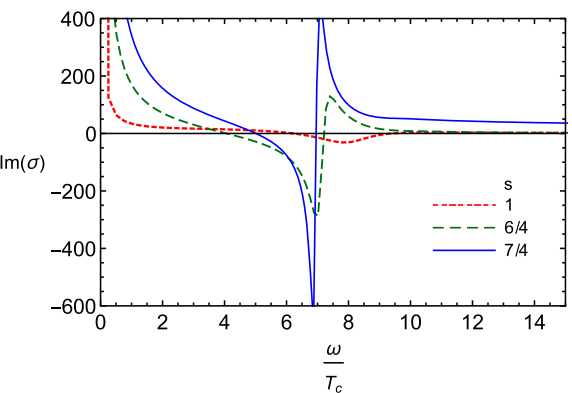

(c) $d=6, T / T_{c} \approx 0.5 T_{c}$

FIG. 5. The imaginary part of conductivity for different $s$ in terms of $\omega$.

depend on the frequency $\omega$ and tends toward a constant value for large frequency [see Figs. 2(b), 2(e) and 2(h)]. At any dimension, for cases $s<d / 4$, at large $\omega$, the conductivity increases with increasing $\omega$ [see Figs. 2(a), 2(d) and 2(g)], and for $s>d / 4$, the conductivity decreases with increasing $\omega$ [see Figs. 2(c), 2(f) and 2(i)]. The fact that in these frequencies, the conductivity dose not change and is a constant for the cases $s=d / 4$, confirms that we have a scale invariant theory for these values of $s$ [54]. In other words, our results is consistent with the conformal field theory which state that the conductivity should reach a constant value. Interestingly enough, the conductivity for this holographic superconductor, at some dimensions and for some values of the power parameter, has an irregular and oscillating behavior at low temperatures, which are shown in Figs. 2(f) and 2(h). The frequency gap is a distinct characteristic of a superconductor, and we are interested in comparing the effects of different values of the power parameter on the behavior of the conductivity and the gap frequency $\omega_{g}$. For this purpose we plot the conductivity in terms of $\omega / T_{c}$ at fixed temperature, but for different values of the power parameter $s$. The results are displayed in Figs. 4 and 5. In [9], it was claimed that for the holographic superconductor the relation $\omega_{g} \approx 8 T_{c}$ is universal. However, it can be observed from the plots that by changing the dimension and power parameter, the frequency gap changes slightly.

\section{THE SUPERCONDUCTOR IN A FINITE MAGNETIC FIELD}

It is well known that the Meissner effect is a general feature of the superconductors, that causes magnetic fields to be expelled from the superconducting region. This means that the superconductor performs a work for expelling the applied magnetic field from the volume, the amount of required work, is proportional to the magnetic field value. We know that the electromagnetic free energy in a superconductor, is minimized and the difference in the free energies between the normal and superconducting phase can provide the energy for this work. If the applied magnetic field is so strong and the difference between the free energies is not enough for expelling the field from the superconductor volume, in this case, the superconductor will exit the superconducting phase and enter the normal phase. At any fixed temperature, the maximum values of the external magnetic field for which above of it, the superconductor cannot have condensation, called as the critical field [5]. Here, we shall investigate the effects of an external magnetic field on the holographic superconductor and would like to find the effect of electrodynamics on the process of expelling the magnetic field. At first, we immerse the superconductor (that is in the normal phase) into an external magnetic field, and then we decrease the temperature until the condensation occurs at a particular temperature. This temperature is the critical 
temperature for that value of the magnetic field, and when we change the value of applied magnetic field, this critical temperature changes, too. The field equations, are the same as in Eqs. (3), (4), and (5), with the difference that in this section we add a magnetic component to the Maxwell field. We consider

$$
A_{a} d x^{a}=\phi(r, x) d t+A_{y}(r, x) d y, \quad \psi=\psi(r, x) .
$$

We rewrite the field equations by considering these assumptions. The original equations in which, the power parameter is a variable, are too long and for the economic reasons we do not present it here. When $s=1$, the equations reduce to the Maxwell form [5],

$$
\begin{aligned}
& \frac{\partial^{2}}{\partial r^{2}} \phi(r, x)+\frac{1}{r^{2} f(r)} \frac{\partial^{2}}{\partial x^{2}} \phi(r, x)-\frac{(d-2)}{r} \frac{\partial}{\partial r} \phi(r, x) \\
& -\frac{2 \phi(r, x) \psi(r, x)^{2}}{f(r)}=0, \\
& \frac{\partial^{2}}{\partial r^{2}} A_{y}(r, x)+\frac{1}{r^{2} f(r)} \frac{\partial^{2}}{\partial x^{2}} A_{y}(r, x)+\frac{(d-4)}{r} \frac{\partial}{\partial r} A_{y}(r, x) \\
& -\frac{2 A_{y}(r, x) \psi(r, x)^{2}}{f(r)}+\frac{\partial f(r) / \partial r}{f(r)} \frac{\partial}{\partial r} A_{y}(r, x)=0, \\
& \frac{\partial^{2}}{\partial r^{2}} \psi(r, x)+\frac{1}{r^{2} f(r)} \frac{\partial^{2}}{\partial x^{2}} \psi(r, x)+\frac{(d-2)}{r} \frac{\partial}{\partial r} \psi(r, x) \\
& -\frac{m^{2} \psi(r, x)}{f(r)}+\frac{\phi(r, x)^{2} \psi(r, x)}{f(r)^{2}}-\frac{A_{y}(r, x)^{2}}{r^{2} f(r)} \\
& \quad+\frac{\partial f(r) / \partial r}{f(r)} \frac{\partial}{\partial r} \psi(r, x)=0 .
\end{aligned}
$$

In the following we study the asymptotic behavior of the field equations. We further assume the scalar field $\psi$ is much smaller than the magnetic field. This is true near the critical temperature when the condensation is formed. Therefore, we treat the scalar field as a perturbation in the bulk and obtain the solution for the equations [38]. In this limit, $\psi$ in Eqs. (29),(30) vanishes, and it is easy to find the solutions. Also, the original versions of these equations with variable $s$ also becomes very simpler by employing perturbative limit. Moreover, we suppose the applied magnetic field has a constant value at the boundary and do not respect the radial coordinate $r$, and $\phi=\phi(r)$. Given these conditions, we find an appropriate solution for the field $A_{y}$ which is $A_{y}=B x$, corresponding to a uniform magnetic field, when $B$ is a constant, that is dual to magnetic field at the boundary. Since our purpose here is to investigate the effects of magnetic field, in the general case. Therefore, we consider the backreaction of the applied magnetic field on the background geometry. In other words, we would like to consider a fully backreacted magnetically charged black hole in the bulk and neglect the backreaction of the scalar field $\psi$ and gauge field $\phi$. Therefore, by solving Eq. (3), we find

$$
f(r)= \begin{cases}r^{2}-\frac{\left(4 r_{+}^{4}+B^{2}\right)}{4 r r_{+}}+\frac{B^{2}}{4 r^{2}}, & \text { for } d=4, \\ r^{2}-\frac{r_{+}^{4}}{r^{2}}+\frac{B^{2}}{6 r^{2}} \ln \left(\frac{r_{+}}{r}\right), & \text { for } d=5, \\ r^{2}-\frac{r_{+}^{5}}{r^{3}}+\frac{B^{2} r_{+}}{8 r^{3}}-\frac{B^{2}}{8 r^{2}}, & \text { for } d=6 .\end{cases}
$$

As before, from the definition of the Hawking temperature $T=\frac{f^{\prime}\left(r_{+}\right)}{4 \pi}$, we find the temperature of this superconductor in the presence of the magnetic field $B$,

$$
T= \begin{cases}\frac{12-B^{2}}{16 \pi}, & \text { for } d=4, \\ \frac{24-B^{2}}{24 \pi}, & \text { for } d=5, \\ \frac{40-B^{2}}{32 \pi}, & \text { for } d=6 .\end{cases}
$$

Inserting $B=0$ in the functions $f(r)$ and $T$, one can obtain the metric function and the Hawking temperature for the higher dimensional Schwarzschild-AdS black holes [4]. With $A_{y}$ and $f(r)$ at hand, one can substitute them in the equation of $\phi$ and $\psi$ and solve these equation numerically. But before that, the equation for $\psi(r, x)=X(x) R(r)$ can be separated, yielding the following differential equations

$$
\begin{aligned}
& R^{\prime \prime}(r)+\left(\frac{f^{\prime}}{f}+\frac{d-2}{r}\right) R^{\prime}(r)+\left(\frac{\phi^{2}(r)}{f^{2}(r)}-\frac{m^{2}}{f(r)}\right) R(r) \\
& \quad=\lambda R(r)
\end{aligned}
$$

when $\lambda$ is the separation constant $\lambda=q n B$ with $n=1$. The solution for $X(x)$, has been discussed in Refs. [5,38].

In order to check the condensation for some values of the applied magnetic field, we solve the field equations for $\phi$ and $\psi$ in the bulk. We expect that the condensation starting point, should be dependent on the values of the magnetic field $B$. Figure 6 , represents the dimensionless condensation with respect to dimensionless temperature, for the conformal invariance case and for a fixed value of the magnetic field $B$. We can see that the dimensionless condensation in the presence of the magnetic field has the same behavior that had in the absence of a magnetic field, but it is clear that the value of this quantity has changed.

Use the results to achieve the critical temperature $T_{c}(B)$. Now we can produce a curve of solutions that have plotted in Fig. 7. It is interesting to plot the dimensionless parameters too. in the presence of power-Maxwell electrodynamics, and for all values of $d$, we find the dimension of $B$, always two.

$T_{c}(0)$ is the critical temperature in the absence of the magnetic field, and $T_{c}(B)$ is the critical temperature in the presence of the magnetic field $B$. In the region that is below the plots, there is superconducting condensation. Actually, 


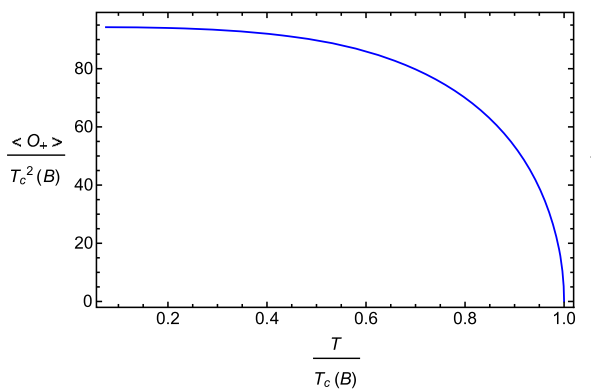

(a) $d=4, s=1, B=1$

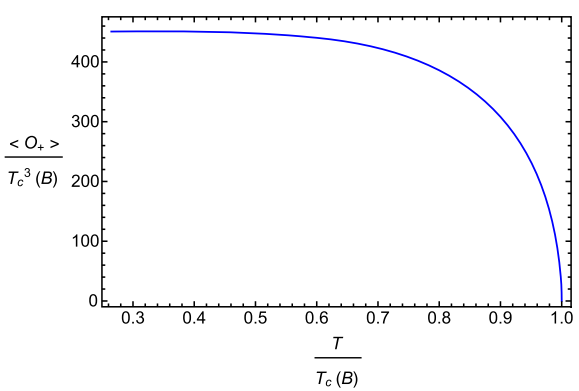

(b) $d=5, s=5 / 4, B=1$

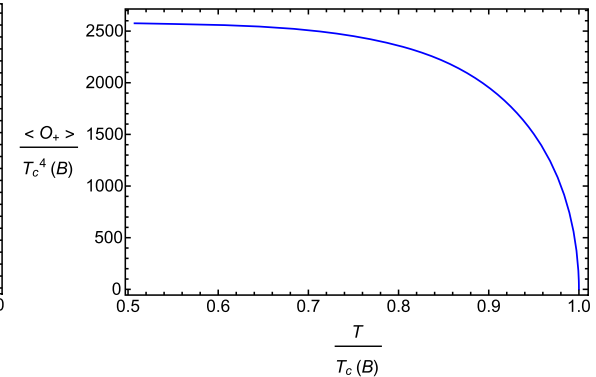

(c) $d=6, s=6 / 4, B=1$

FIG. 6. The dimensionless condensation in the presence of external magnetic field versus temperature.

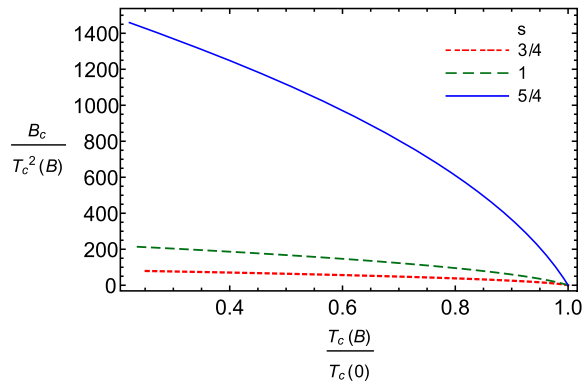

(a) $d=4$

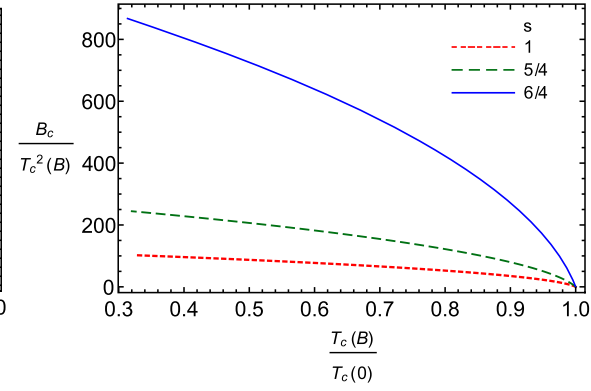

(b) $d=5$

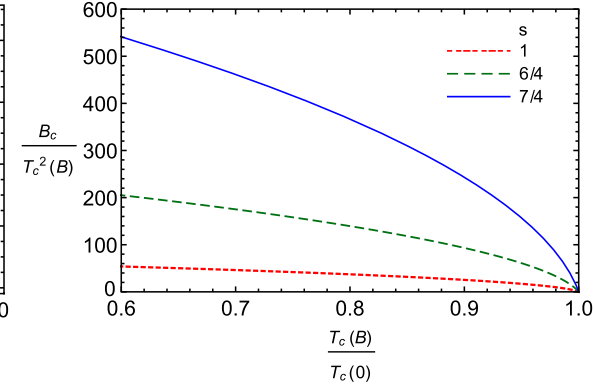

(c) $d=6$

FIG. 7. The critical magnetic field versus temperature.

the below region, displays all values of temperature and simultaneously, the values of the critical magnetic field for which the superconductor remains in the superconducting phase. As we grow up the temperature or the applied magnetic field, depending on the spacetime dimension and the power parameter, the superconductor leaves the superconducting phase at a point (on the lines of the plots). We can also see that for all values of $s$ and $d$, as the magnetic field increases, the critical temperature decreases, so the condensation becomes harder. This is an expected result, which can be interpreted as the Meissner-like effect for holographic superconductors.

\section{CLOSING REMARKS}

In this paper, based on the numerical shooting method, we have explored the holographic $s$-wave superconductor in the presence of power-Maxwell electrodynamics and in all higher dimensions. We have derived the critical temperature for this kind of superconductor and found out that it decreases with increasing the power parameter $s$, which means it is harder for the condensation to form in the presence of stronger electrodynamics. We observed that when the power parameter is smaller that one $(s<1)$ the critical temperature increases as well [49]. In this case the strength of the gauge field decreases for the powerMaxwell case comparing to the Maxwell case $(s=1)$. Therefore, the effects of the gauge field on the background decrease as well. This implies that it is quite reasonable to take still the probe limit when the gauge field is in the form of the power-Maxwell electrodynamics. The obtained results are also consistent with the fact that in higher dimensional systems, the phase transition are easier to achieve. We have obtained the condensation value and the critical exponent and showed that for all values of the power parameter $s$ and dimension $d$, the critical exponent is $1 / 2$. We observed that in higher dimensions and for larger value of $s$, the rescaled condensation operator increase, explicitly. We explored the electrical conductivity by applying an external electric field to the system. In this regards, we obtained infinite dc conductivity for this superconductor and showed that the superconducting gap appeared and becomes deep with decreasing the temperature. We observed that at enough large frequency, for a fixed dimension of spacetime $d$, and for the conformal invariance power-Maxwell Lagrangian $s=d / 4$, the conductivity does not depend on the frequency $\omega$ and tends toward a constant value for large frequency. This is consistent with the fact that in conformal field theory, the conductivity reaches a constant value at large frequency. We also investigated the cases with $s<d / 4$ and $s>d / 4$, separately. In the former case, the conductivity increases with increasing $\omega$, while in the latter one the conductivity decreases with increasing $\omega$. Also, the ratio $\omega_{g} / T_{c}$ varies slightly with changing the dimension and power parameter. We have also immersed the superconductor into an 
external magnetic field and studied the behavior of the critical magnetic field. We found out that for all value of $d$ and $s$, the magnetic field has an expected behavior. As the magnetic field increases, the critical temperature decreases, so the condensation becomes harder. This can be interpreted as the Meissner-like effect for holographic superconductors.

\section{ACKNOWLEDGMENTS}

We thank Shiraz University Research Council. A. S. is grateful to the University of Oldenburg, for hospitality. The work of A. S. has been supported financially by Research Institute for Astronomy and Astrophysics of Maragha (RIAAM), Iran.
[1] J. Maldacena, The large N limit of superconformal field theories and supergravity, Adv. Theor. Math. Phys. 2, 231 (1998).

[2] S. S. Gubser, I. R. Klebanov, and A. M. Polyakov, Gauge theory correlators from non-critical string theory, Phys. Lett. B 428, 105 (1998).

[3] E. Witten, Anti de sitter space and holography, Adv. Theor. Math. Phys. 2, 253 (1998).

[4] S. A. Hartnoll, C. P. Herzog, and G. T. Horowitz, Building a Holographic Superconductor, Phys. Rev. Lett. 101, 031601 (2008).

[5] S. A. Hartnoll, C. P. Herzog, and G. T. Horowitz, Holographic superconductors, J. High Energy Phys. 12 (2008) 015.

[6] S. S. Gubser, Breaking an Abelian gauge symmetry near a black hole horizon, Phys. Rev. D 78, 065034 (2008).

[7] C. P. Herzog, Lectures on holographic superfluidity and superconductivity, J. Phys. A 42, 343001 (2009).

[8] S. A. Hartnoll, Lectures on holographic methods for condensed matter physics, Classical Quantum Gravity 26, 224002 (2009).

[9] G. T. Horowitz and M. M. Roberts, Holographic superconductors with various condensates, Phys. Rev. D 78, 126008 (2008).

[10] G. T. Horowitz, Introduction to holographic superconductors, Lect. Notes Phys. 828, 313 (2011).

[11] Q. Pan, J. Jing, B. Wang, and S. Chen, Analytical study on holographic superconductors with backreactions, J. High Energy Phys. 06 (2012) 087.

[12] H. B. Zeng, X. Gao, Y. Jiang, and H. S. Zong, Analytical computation of critical exponents in several holographic superconductors, J. High Energy Phys. 05 (2011) 002.

[13] R. G. Cai, H. F. Li, and H. Q. Zhang, Analytical studies on holographic insulator/superconductor phase transitions, Phys. Rev. D 83, 126007 (2011).

[14] Q. Pan, B. Wang, E. Papantonopoulos, J. Oliveira, and A. B. Pavan, Holographic superconductors with various condensates in Einstein-Gauss-Bonnet gravity, Phys. Rev. D 81, 106007 (2010).

[15] Q. Pan and B. Wang, General holographic superconductor models with Gauss-Bonnet corrections, Phys. Lett. B 693, 159 (2010).

[16] H. F. Li, R. G. Cai, and H. Q. Zhang, Analytical studies on holographic superconductors in Gauss-Bonnet gravity, J. High Energy Phys. 04 (2011) 028.
[17] S. L. Cui and Z. Xue, Critical magnetic field in holographic superconductor in Gauss-Bonnet gravity with Born-Infeld electrodynamics, Phys. Rev. D 88, 107501 (2013).

[18] L. Barclay, R. Gregory, S. Kanno, and P. Sutcliffe, GaussBonnet holographic superconductors, J. High Energy Phys. 12 (2010) 029.

[19] D. Momeni, M. Raza, and R. Myrzakulov, Holographic superconductors with Weyl corrections, Int. J. Geom. Methods Mod. Phys. 13, 1550131 (2016).

[20] R. Gregory, S. Kanno, and J. Soda, Holographic superconductors with higher curvature corrections, J. High Energy Phys. 10 (2009) 010.

[21] X. M. Kuang, E. Papantonopoulos, G. Siopsis, and B. Wang, Building a holographic superconductor with higherderivative couplings, Phys. Rev. D 88, 086008 (2013).

[22] R. G. Cai, H. F. Li, and H. Q. Zhang, Analytical studies on holographic insulator/superconductor phase transitions, Phys. Rev. D 83, 126007 (2011).

[23] A. Sheykhi and F. Shaker, Analytical study of holographic superconductor in Born-Infeld electrodynamics with backreaction, Phys. Lett. B 754, 281 (2016).

[24] S. Gangopadhyay and D. Roychowdhury, Analytic study of properties of holographic superconductors in Born-Infeld electrodynamics, J. High Energy Phys. 05 (2012) 002.

[25] A. Sheykhi and F. Shaker, Effects of backreaction and exponential nonlinear electrodynamics on the holographic superconductors, Int. J. Mod. Phys. D 26, 1750050 (2017).

[26] D. Ghorai and S. Gangopadhyay, Higher dimensional holographic superconductors in Born-Infeld electrodynamics with backreaction, Eur. Phys. J. C 76, 146 (2016).

[27] C. Lai, Q. Pan, J. Jing, and Y. Wang, On analytical study of holographic superconductors with Born-Infeld electrodynamics, Phys. Lett. B 749, 437 (2015).

[28] J. Jing and S. Chen, Holographic superconductor in the Boren-Infeld electrodynamics, Phys. Lett. B 686, 68 (2010).

[29] J. Jing, Q. Pan, and S. Chen, Holographic superconductor/ insulator transition with logarithmic electromagnetic field in Gauss-Bonnet gravity, Phys. Lett. B 716, 385 (2012).

[30] H. B. Zeng, Y. Tian, Z. Fan, and C. M. Chen, Nonlinear conductivity of a holographic superconductor under constant electric field, Phys. Rev. D 95, 046014 (2017).

[31] R. A. Konoplya and A. Zhidenko, Holographic conductivity of zero temperatur superconductors, Phys. Lett. B 686, 199 (2010). 
[32] A. Sheykhi, D. H. Asl, and A. Dehyadegari, Conductivity of higher dimensional holographic superconductors with nonlinear electrodynamics, Phys. Lett. B 781, 139 (2018).

[33] A. Sheykhi, A. Ghazanfari, and A. Dehyadegari, Holographic conductivity of holographic superconductors with higher order corrections, Eur. Phys. J. C 78, 159 (2018).

[34] B. B. Ghotbabadi, M. K. Zangeneh, and A. Sheykhi, Onedimensional backreacting holographic superconductors with exponential nonlinear electrodynamics, Eur. Phys. J. C 78, 381 (2018).

[35] M. Mohammadi, A. Sheykhi, and M. Kord Zangeneh, Analytical and numerical study of backreacting one-dimensional holographic superconductors in the presence of BornInfeld electrodynamics, Eur. Phys. J. C 78, 654 (2018).

[36] D. Ghorai and S. Gangopadhyay, Conductivity of holographic superconductors in Born-Infeld electrodynamics, Nucl. Phys. B933, 1 (2018).

[37] E. Nakano and W. Y. Wen, Critical magnetic field in AdS/ CFT superconductor, Phys. Rev. D 78, 046004 (2008).

[38] T. Albash and C. V. Johnson, A holographic superconductor in an external magnetic field, J. High Energy Phys. 09 (2008) 121.

[39] D. Momeni, E. Nakano, M. R. Setare, and Wen-Yu Wen, Analytical study of critical magnetic field in a holographic superconductor, Int. J. Mod. Phys. A 28, 1350024 (2013).

[40] X. H. Ge, B. Wang, S. F. Wu, and G. H. Yang, Analytical study on holographic superconductors in external magnetic field, J. High Energy Phys. 08 (2010) 108.

[41] D. Roychowdhury, AdS/CFT superconductors with power Maxwell electrodynamics: Reminiscent of the Meissner effect, Phys. Lett. B 718, 1089 (2013).

[42] S. L. Cui and Z. Xue, Critical magnetic in holographic superconductor in Gauss-Bonnet gravity with Born-Infeld electrodynamics, Phys. Rev. D 88, 107501 (2013).

[43] Z. Zhao, Q. Pan, and J. Jing, Notes on analytical study of holographic superconductors with Lifshitz scaling in external magnetic field, Phys. Lett. B 735, 438 (2014).
[44] A. Sheykhi and F. Shamsi, Holographic superconductors with Logarithmic nonlinear electrodynamics in an external magnetic field, Int. J. Theor. Phys. 56, 916 (2017).

[45] M. Hassaine and C. Martinez, Higher-dimensional black holes with a conformally invariant Maxwell source, Phys. Rev. D 75, 027502 (2007).

[46] A. Sheykhi, Higher dimensional charged $f(R)$ black holes, Phys. Rev. D 86, 024013 (2012).

[47] J. Jing, Q. Pan, and S. Chen, Holographic superconductors with power-Maxwell field, J. High Energy Phys. 11 (2011) 045 .

[48] J. Jing, L. Jiang, and Q. Pan, Holographic superconductors for the power-Maxwell field with backreactions, Classical Quantum Gravity 33, 025001 (2016).

[49] A. Sheykhi, H. R. Salahi, and A. Montakhab, Analytical and numerical study of Gauss-Bonnet holographic superconductors with power-Maxwell field, J. High Energy Phys. 04 (2016) 058.

[50] A. Sheykhi, H. R. Salahi, and A. Montakhab, Effects of backreaction on power-Maxwell holographic superconductors in Gauss-Bonnet gravity, Eur. Phys. J. C 76, 575 (2016).

[51] A. Sheykhi, F. Shamsi, and S. Davatolhagh, The upper critical magnetic field of holographic superconductor with conformally invariant power-Maxwell electrodynamics, Can. J. Phys. 95, 450 (2017).

[52] A. V. Ramallo, Introduction to the AdS/CFT correspondence, Springer Proc. Phys. 161, 411 (2015).

[53] J. Bardeen, L. N. Cooper, and J. R. Schrieer, Theory of superconductivity, Phys. Rev. 108, 1175 (1957).

[54] D. Tong, Lectures on holographic conductivity, Cracow School of Theoretical Physics (2013), http://www.damtp .cam.ac.uk/user/tong/talks/zakopane.pdf.

[55] K. Skenderis, Lecture note on holographic renormalization, Classical Quantum Gravity 19, 5849 (2002). 